\title{
Preliminary study on Anti-inflammatory and Anti-tumor Effects of Gelatin - based on pH-responsive Drug- loaded Nanoparticles
}

Fen Lin*

Shanghai East Foreign Language School Affiliated to SISU

\begin{abstract}
Gelatin as one kind of nano-drug carrier is a polysaccharide which can be absorbed after releasing all nano-drugs. Poly(ethylene glycol) as a polymer material that can modify nano-drugs is able to extend circulatory time of doxorubicin and aspirin, enhance stability and reduce toxicity. At precancerous, aspirin will be released slowly to resist inflammation. When tumor cell appears at a more advance stage, doxorubicin release from Gel to achieve anti-tumor effect. After all those steps, poly (ethylene glycol) could be expelled from body.

In brief, the nano-drug that has doxorubicin and aspirin is able to prevent the growth of tumor cells and kill them when they appear more precisely.
\end{abstract}

Publication date: March, 2019

Publication online: 31 March, 2019

*Corresponding author: Fen Lin, liaoquanneng@ ivygate.cn

\section{Introduction}

Over the past decades, nanomedicines based on polymers have been intensively studied for cancer therapy, regarding their potential to increase drug solubility, enhance therapeutic effect and reduce side effects. Comparing to small molecular anticancer drugs, nanomedicines have shown many advantages including prolonged circulation time by evading glomerular filtration, improved pharmacokinetic properties, as well as enhanced tumor accumulation via the enhanced permeation and retention (EPR) effect. Among various nanomedicines such as polymeric nanoparticles, prodrugs, micelles, vesicles, nanogels and liposomes, prodrug-based nanoparticles have drawn much more attention due to the clear and simple structure and great potential in clinical translation.

Adriamycin, a new antineoplastic drug, has a good therapeutic effect on many tumors and can inhibit the growth of tumors greatly, such as solid tumors and hematological malignancies. Although adriamycin has some side effects, its efficacy is better than that of similar drugs. Therefore, it can achieve good therapeutic effect in small doses. Adriamycin therapy results in dose-limiting hematologic and cardiac toxicity, as well as stomatitis, nausea, vomiting, and alopecia, but in general these toxicities are predictable and reversible.

Gelatin is a commonly used natural polymer which is derived from collagen. The isoelectric point of gelatin can be modified during the fabrication process to yield either a negatively charged acidic gelatin, or a positively charged basic gelatin at physiological pH. This theoretically allows electrostatic interactions to take place between a charged biomolecule and gelatin of the opposite charge, forming polyion complexes. Various forms of gelatin carrier matrices can be fabricated for controlled release studies, and characterization studies have been performed which show that gelatin carriers are able to sorb charged biomolecules such as proteins and plasmid DNA through polyion complexation. The crosslinking density of gelatin hydrogels has been shown to affect their degradation rate in vivo, and the rate of biomolecule release from gelatin carriers has been shown to have a similar profile, suggesting that complexed gelatin/biomolecule fragments are released by enzymatic degradation of the carrier in vivo. 


\section{Experimental verification}

\subsection{Preparation of nano drugs}

1. $150 \mathrm{mg}$ of HO-PEG-OH, $36 \mathrm{mg}$ of aspirin, $4.88 \mathrm{mg}$ of EDCI and $42.08 \mathrm{mg}$ of DMAP were dissolved in 50 $\mathrm{ml}$ of dichloromethane for $24 \mathrm{~h}$, extracted three times with saturated brine, dehydrated with $\mathrm{MgSO} 4$, filtered, and evaporated to remove the solvent. The drug was a white solid and the white solid was recorded as the sample PEG-ASP.

2. Take $50 \mathrm{mg}$ PEG-CHO, $25 \mathrm{mg}$ deionized doxorubicin dissolved in $35 \mathrm{ml}$ TEA for $48 \mathrm{~h}$, remove the reaction solvent by rotary evaporation, the drug is red solid, record the red solid as sample PEG-DOX.

\subsection{Measuring sample PEG-ASP and sample PEG-DOX particle size and nuclear magnetic}

1.Take $10 \mathrm{mg}$ of sample PEG-ASP, dissolve it in 10 $\mathrm{ml}$ of pure water, prepare a PEG-ASP solution at a concentration of $1 \mathrm{mg} / \mathrm{ml}$, and measure the particle size and nuclear magnetic properties of the sample. The reaction was carried out for 12 hours by adding $\mathrm{NaOH}$, and the particle diameter of the sample after the reaction was measured. As shown in Figure 1, it can be seen from the nuclear magnetic map that HO-PEG-OH reacts with aspirin to synthesize a new substance.

2.Take $10 \mathrm{mg}$ of sample PEG-DOX, dissolve it in $10 \mathrm{ml}$ of pure water, prepare a PEG-DOX solution at a concentration of $1 \mathrm{mg} / \mathrm{ml}$, and measure the particle size and nuclear magnetic properties of the sample. The reaction was carried out for $30 \mathrm{~min}$, and the particle size of the sample after the reaction was measured. As shown in Figure 2, it can be seen from the nuclear magnetic diagram that PEG-CHO and doxorubicin have produced new substances.

\subsection{Production of aspirin standard curve}

Take $10 \mathrm{mg}$ of aspirin and dissolve it in $10 \mathrm{ml}$ of aqueous solution to prepare aspirin mother liquor at a concentration of $1 \mathrm{mg} / \mathrm{ml}$, and dilute some of the mother liquor to obtain solution concentrations of $0.5 \mathrm{mg} / \mathrm{ml}$, $0.1 \mathrm{mg} / \mathrm{ml}, 0.01 \mathrm{mg} / \mathrm{ml}, 0.001 \mathrm{mg} / \mathrm{ml}$. The absorbance values of five different concentrations of aspirin solution were determined separately. The absorbance is the abscissa and the corresponding concentration is the ordinate. A standard curve is produced.

\subsection{Production of doxorubicin standard curve}

Take $10 \mathrm{mg}$ of doxorubicin and dissolve it in $10 \mathrm{ml}$ of aqueous solution to prepare doxorubicin mother liquor at a concentration of $1 \mathrm{mg} / \mathrm{ml}$, and dilute some of the mother liquor to obtain solution concentrations of $0.5 \mathrm{mg} / \mathrm{ml}, 0.1 \mathrm{mg} / \mathrm{ml}, 0.01 \mathrm{mg} / \mathrm{ml}, 0.001 \mathrm{mg} / \mathrm{ml}$. The absorbance values of five different concentrations of doxorubicin solution were determined separately. The absorbance is the abscissa and the corresponding concentration is the ordinate. A standard curve is produced.

\subsection{Preparation of PEG-ASP colloid and its release in ethanol}

Take $0.5 \mathrm{mg}$ sample PEG-ASP dissolved in $1 \mathrm{ml}$ absolute ethanol, add $4 \mathrm{ml}$ pure water, the solution concentration is $0.1 \mathrm{mg} / \mathrm{ml}$, add $750 \mathrm{mg}$ gelatin, heat to shake to dissolve, put it into the refrigerator to freeze and solidify, remove the solidified colloid, cut A small portion was observed under electron microscope for the morphology of the drug-loaded colloid, as shown in Figure 5. The remaining colloids were immersed in pure ethanol, and the absorbance of the samples were measured at $1 \mathrm{~h}, 2 \mathrm{~h}, 4 \mathrm{~h}, 8 \mathrm{~h}, 12 \mathrm{~h}$, and $24 \mathrm{~h}$. The aspirin concentration in ethanol was calculated from the standard curve of aspirin for $1 \mathrm{~h}, 2 \mathrm{~h}, 4 \mathrm{~h}, 8 \mathrm{~h}, 12 \mathrm{~h}$, and $24 \mathrm{~h}$. The reaction was carried out by adding $\mathrm{NaOH}$ for 12 hours, and the absorbance was measured to obtain an aspirin concentration. As shown in Figure 7, it can be seen that as the concentration of aspirin increases, the amount of colloidal drug release increases. When the concentration of aspirin is less than $20 \mathrm{mg} / \mathrm{ml}$, the release amount increases with the increase of aspirin concentration and increases rapidly. When the concentration of aspirin is greater than $20 \mathrm{mg} / \mathrm{ml}$, the release amount increases with the increase of aspirin concentration, but the growth rate gradually decreases.

\subsection{Preparation of PEG-DOX colloid and its release in ethanol}

Take $0.5 \mathrm{mg}$ sample PEG-DOX dissolved in $1 \mathrm{ml}$ absolute ethanol, add $4 \mathrm{ml}$ pure water, the solution concentration is $0.1 \mathrm{mg} / \mathrm{ml}$, add $750 \mathrm{mg}$ gelatin, heat to shake to dissolve, put it into the refrigerator to freeze and solidify, take out the solidified colloid, soak In pure ethanol, the absorbance of the samples was measured at $1 \mathrm{~h}, 2 \mathrm{~h}$, $4 \mathrm{~h}, 8 \mathrm{~h}, 12 \mathrm{~h}$, and $24 \mathrm{~h}$, respectively. The doxorubicin concentration in ethanol was calculated from the standard curve of aspirin for $1 \mathrm{~h}, 2 \mathrm{~h}, 4 \mathrm{~h}, 8 \mathrm{~h}, 12 \mathrm{~h}$, and $24 \mathrm{~h}$. The reaction was carried out for $30 \mathrm{~min}$ by adding $\mathrm{HCl}$, and the absorbance was measured to obtain the concentration 
of doxorubicin. As shown in Figure 8, it can be seen that the amount of doxorubicin released gradually increased with increasing doxorubicin concentration before the addition of $\mathrm{HCl}$. After the addition of $\mathrm{HCl}$, as the concentration of doxorubicin increased, the release amount increased rapidly.

\subsection{Preparation of PEG-ASP and PEG-DOX mixed colloids and their release in ethanol}

Take $0.5 \mathrm{mg}$ sample PEG-ASP and $1.315 \mathrm{mg}$ sample PEG-DOX dissolved in $1 \mathrm{ml}$ absolute ethanol, add 4 $\mathrm{ml}$ pure water, the solution concentration is $0.1 \mathrm{mg} /$ $\mathrm{ml}$, add $750 \mathrm{mg}$ gelatin, heat to shake to dissolve, put into the refrigerator to freeze and solidify, The solidified colloid was taken out and immersed in pure ethanol. The absorbance of the sample was measured at $1 \mathrm{~h}, 2 \mathrm{~h}, 4 \mathrm{~h}, 8 \mathrm{~h}, 12 \mathrm{~h}$, and $24 \mathrm{~h}$, respectively. The drug concentration of PEG-DOX and PEG-ASP mixed colloid was obtained. After adding $\mathrm{NaOH}$ for 12 hours, $\mathrm{HCl}$ was added for $30 \mathrm{~min}$, and the colloidal absorbance was measured to obtain the concentration of the drug released by the colloid after the chemical reaction bond was broken.

\section{Conclusions}

This research aims to solve the existing problems by combining synthesized drugs of low biotoxicity and high $\mathrm{pH}$ sensitivity with nano-drug carriers, which not only do little harm but also stay longer inside the body to release drug when available.

1. In brief, the nano-drug that has doxorubicin and aspirin is able to prevent the growth of tumor cells and kill them when they appear more precisely.

2. The invention lies in the field of nanomedicine. It is able to release anti-inflammatory drug and antitumor drug according two different environments: the inflammatory condition in early stage of cancers, and the acid condition which has specific $\mathrm{pH}$ value after appearing of tumour cells. 